\title{
Angiogenesis induced by prenatal ischemia predisposes to periventricular hemorrhage during postnatal mechanical ventilation
}

\author{
Cigdem Tosun' ', Caron Hong'², Brianna Carusillo', Svetlana Ivanova', Volodymyr Gerzanich¹ and J. Marc Simard ${ }^{1,3,4}$
}

BACKGROUND: Three risk factors are associated with hemorrhagic forms of encephalopathy of prematurity (EP): (i) prematurity, (ii) in utero ischemia (IUI) or perinatal ischemia, and (iii) mechanical ventilation. We hypothesized that IUI would induce an angiogenic response marked by activation of vascular endothelial growth factor (VEGF) and matrix metalloproteinase-9 (MMP-9), the latter degrading vascular basement membrane and increasing vulnerability to raised intravenous pressure during positive pressure mechanical ventilation.

METHODS: We studied a rat model of hemorrhagic-EP characterized by periventricular hemorrhages in which a 20-min episode of IUI is induced at E19, pups are born naturally at E2122, and on P0, are subjected to a 20-min episode of positive pressure mechanical ventilation. Tissues were studied by H\&E staining, immunolabeling, immunoblot, and zymography.

RESULTS: Mechanical ventilation of rat pups 2-3 d after 20-min IUI caused widespread hemorrhages in periventricular tissues. IUI resulted in upregulation of VEGF and MMP-9. Zymography confirmed significantly elevated gelatinase activity. MMP-9 activation was accompanied by severe loss of MMP-9 substrates, collagen IV and laminin, in microvessels in periventricular areas.

CONCLUSION: Our findings are consistent with the hypothesis that positive pressure mechanical ventilation of the newborn in the context of recent prenatal ischemia/hypoxia can predispose to periventricular hemorrhages.

\section{INTRODUCTION}

Encephalopathy of prematurity (EP) is a broad designation that encompasses numerous neurological disorders of early childhood, including nonhemorrhagic lesions such as periventricular leukomalacia, hemorrhagic lesions such as choroid plexus, germinal matrix and other periventricular hemorrhages that may extend as intraventricular hemorrhages, various neuronal, axonal and oligodendrocyte pathologies, and hydrocephalus $(1,2)$. Infants who survive may suffer from cognitive, behavioral and motor (cerebral palsy) abnormalities that persist for life.
In human infants, hemorrhages into the ventricles and into periventricular tissues are categorized into four major grades, based on the presence of intraventricular hemorrhage (IVH) or of parenchymal periventricular hemorrhage ( $\mathrm{PVH})$ : IVH1-3 are germinal matrix hemorrhages that either do not (IVH1) or that do (IVH2 and IVH3) extend into the ventricles; PVH1 refers to periventricular venous infarction(s) associated with parenchymal hemorrhage plus variable amounts (small, moderate or large) of IVH (3). IVH3 and PVH1 may have different etiologies and different long-term neurodevelopmental outcomes, but both are considered "severe." Overall, the grade of hemorrhage predicts long-term clinical outcome (4). Previous brain hemorrhages in premature infants are associated with later white matter abnormalities on magnetic resonance imaging (5), with the topography and severity of periventricular white matter lesions believed to have long-term predictive value for cognitive and social capabilities in survivors of preterm birth (6).

Apart from prematurity, analyses of clinical cases have shown that two other risk factors are associated with brain hemorrhages: (i) in utero or perinatal ischemia/hypoxia, and (ii) early postnatal mechanical ventilation $(7,8)$. The use of positive pressure mechanical ventilation in premature infants has been associated with cerebral palsy, delayed neurological development, and other neurological disorders typical of EP (9-11).

Animal models have been used successfully to replicate germinal matrix hemorrhages (IVH1-3), and have demonstrated the critical role of angiogenesis in the premature germinal matrix predisposing to hemorrhage $(12,13)$. Less attention has been directed toward modeling parenchymal hemorrhages involving periventricular tissues. We recently described a rat model that is characterized by hemorrhages in periventricular tissues, including choroid plexus, subventricular zone, hippocampus, and periventricular white matter $(14,15)$. The model encompasses the tandem insults of $20 \mathrm{~min}$ of intrauterine ischemia (IUI) at the gestational age of embryonic day 19 (E19), followed 2-3 d later, 6-8h after natural birth, with an intraperitoneal injection of glycerol. Rats subjected to these tandem insults perinatally later exhibit, as adolescents, significant 
developmental delay and abnormal cognitive and motor performance. The IP injection of glycerol, which was shown to raise intravenous pressure, was found to precipitate venous hemorrhages, especially in periventricular tissues. However, the molecular mechanism for the increase in susceptibility to raised intravenous pressure induced by IUI was not examined, and the use of glycerol to raise intravenous pressure lacked clinical relevance.

The matrix metalloproteinase, MMP-9, is a secreted zincdependent extracellular endopeptidase that degrades components of the vascular basement membrane, including collagen IV, laminin, elastin, and fibronectin (16). In the adult brain, MMP-9 is upregulated/activated by cerebral ischemia/reperfusion, where it actively contributes to the formation of vasogenic edema, and where it is causally implicated in hemorrhagic transformation following adult stroke (17). In the neonatal brain, upregulation of MMP-9, along with vascular endothelial growth factor (VEGF), forms a crucial part of the angiogenic response to ischemia, with MMP-9 serving the important role of proteolytic matrix degradation that is needed for the migration of endothelial cells and vascular remodeling (18). As in the adult, MMP-9 activation in the neonatal brain promotes blood-brain barrier leakage, vasogenic edema and other deleterious effects (19-21).

We hypothesized that prenatal ischemia might induce an angiogenic response that would be accompanied by upregulation of VEGF (22) and MMP-9, the latter causing degradation of immature vascular basement membrane proteins, diminished structural integrity of vessels, and increased vulnerability to raised intravenous pressure, as can occur with positive pressure mechanical ventilation (23). Here, we show that $20 \mathrm{~min}$ of mechanical ventilation of newborn rat pups 2-3 $\mathrm{d}$ after IUI causes widespread hemorrhages in periventricular tissues. Our data indicate that IUI results in a significant angiogenic response, with upregulation of VEGF and MMP-9 that is accompanied by loss of the MMP-9 substrates, basal lamina type IV collagen and laminin. Together, our findings are consistent with the hypothesis that positive pressure mechanical ventilation of the newborn in the context of recent prenatal ischemia/hypoxia can predispose to $\mathrm{PVH}$.

\section{RESULTS}

\section{Blood gases and electrolytes}

We measured blood gases and electrolytes in mixed arterial/ venous blood of pups that were mechanically ventilated, and compared them to values from pups with spontaneous ventilation. Samples were collected $6 \mathrm{~h}$ after birth, and in the case of ventilated pups, immediately after the completion of $20 \mathrm{~min}$ of ventilation. There were no significant differences in blood gases or electrolytes between the two groups (Table 1), and values were similar to those previously reported in rat pups $4 \mathrm{~h}$ after birth (24).

\section{Brain hemorrhages}

Coronal brain sections stained with $\mathrm{H} \& \mathrm{E}$ were used to document brain hemorrhages in pups that were mechanically
Table 1. Mixed arterial/venous blood gases and electrolytes in unventilated vs. mechanically ventilated newborn pups $6 \mathrm{~h}$ after birth $^{\mathrm{a}}$

\begin{tabular}{lcrc}
\hline & Nonventilated & Ventilated & $p^{\mathrm{b}}$ \\
\hline $\mathrm{pO}_{2}(\mathrm{mmHg})$ & $82.2 \pm 6.8$ & $80.8 \pm 7.9$ & 0.91 \\
$\mathrm{pCO}_{2}(\mathrm{mmHg})$ & $65.5 \pm 6.2$ & $71.7 \pm 3.8$ & 0.37 \\
$\mathrm{pH}$ & $7.16 \pm 0.03$ & $7.12 \pm 0.02$ & 0.24 \\
Bicarbonate $(\mathrm{mmol} / \mathrm{l})$ & $24.0 \pm 1.0$ & $21.7 \pm 0.8$ & 0.11 \\
$\mathrm{CSO}_{2}$ & $89 \pm 4.1$ & $83.1 \pm 3.0$ & 0.27 \\
Sodium $(\mathrm{mmol} / \mathrm{l})$ & $132.7 \pm 0.6$ & $132.6 \pm 0.6$ & 0.88 \\
Potassium $(\mathrm{mmol} / \mathrm{l})$ & $5.0 \pm 0.4$ & $4.3 \pm 0.4$ & 0.33 \\
Chloride $(\mathrm{mmol} / \mathrm{l})$ & $103.6 \pm 0.7$ & $102.8 \pm 0.7$ & 0.47 \\
Concentration total $\mathrm{CO}_{2}$ & $25.9 \pm 1.1$ & $24.0 \pm 0.9$ & 0.20 \\
Anion gap & $11.5 \pm 1.0$ & $12.2 \pm 0.5$ & 0.52 \\
Hematocrit & $18.1 \pm 1.3$ & $18.8 \pm 0.8$ & 0.67 \\
Hgb & $6.1 \pm 0.4$ & $6.4 \pm 0.3$ & 0.61 \\
Glucose & $75.5 \pm 3.5$ & $77.9 \pm 2.8$ & 0.61 \\
Lactate & $1.7 \pm 0.1$ & $2.1 \pm 0.2$ & 0.08 \\
\hline aMean \pm SE; data from 10 nonventilated pups (pooled from 2 sham-injured, $8 \mathrm{IUI})$ and \\
from 18 ventilated pups (pooled from 6 sham-injured and 12 IUI). bUnpaired t-test, \\
two-tailed.
\end{tabular}

ventilated and had been subjected to either IUI or sham-injury in utero. Pups subjected to $20 \mathrm{~min}$ IUI plus $20 \mathrm{~min}$ of mechanical ventilation after birth (IUI+ventilation group) showed prominent hemorrhages in periventricular tissues, including choroid plexus, lateral ventricle, subventricular zone, hippocampus, and periventricular white matter, as well as in cortex (Figure 1a-h) (14,15). In some cases, large hemorrhages were observed in the fibria, corpus callosum, and cortex. By contrast, mechanical ventilation in pups subjected to sham-injury in utero (sham+ventilation group) resulted in only rare punctate hemorrhages, mainly in the hippocampus.

Analysis of the distribution of the hemorrhages confirmed the high frequency as well as the predominant periventricular location in pups subjected to the dual insults, compared to controls with sham-injury in utero and mechanical ventilation after birth (Figure 2). The minimal hemorrhages in the control pups (sham+ventilation group) resembled the minimal hemorrhages previously observed with 20-min IUI alone at the same gestational age (no postnatal insult) (14). Thus, neither insult alone appeared to be particularly harmful, but both combined-IUI plus postnatal ventilation-yielded significant PVH.

\section{Loss of collagen IV and laminin at birth}

The susceptibility to PVH in pups subjected to IUI plus postnatal ventilation suggested that the structural integrity of microvessels might have been compromised secondary to IUI. The structural integrity of microvessels is determined by several factors, including the integrity and composition of the basement membrane, a crucial element of which is collagen IV (25).

We evaluated collagen IV in the brain at different times after IUI, including in fetuses 3-24h after IUI, and in 

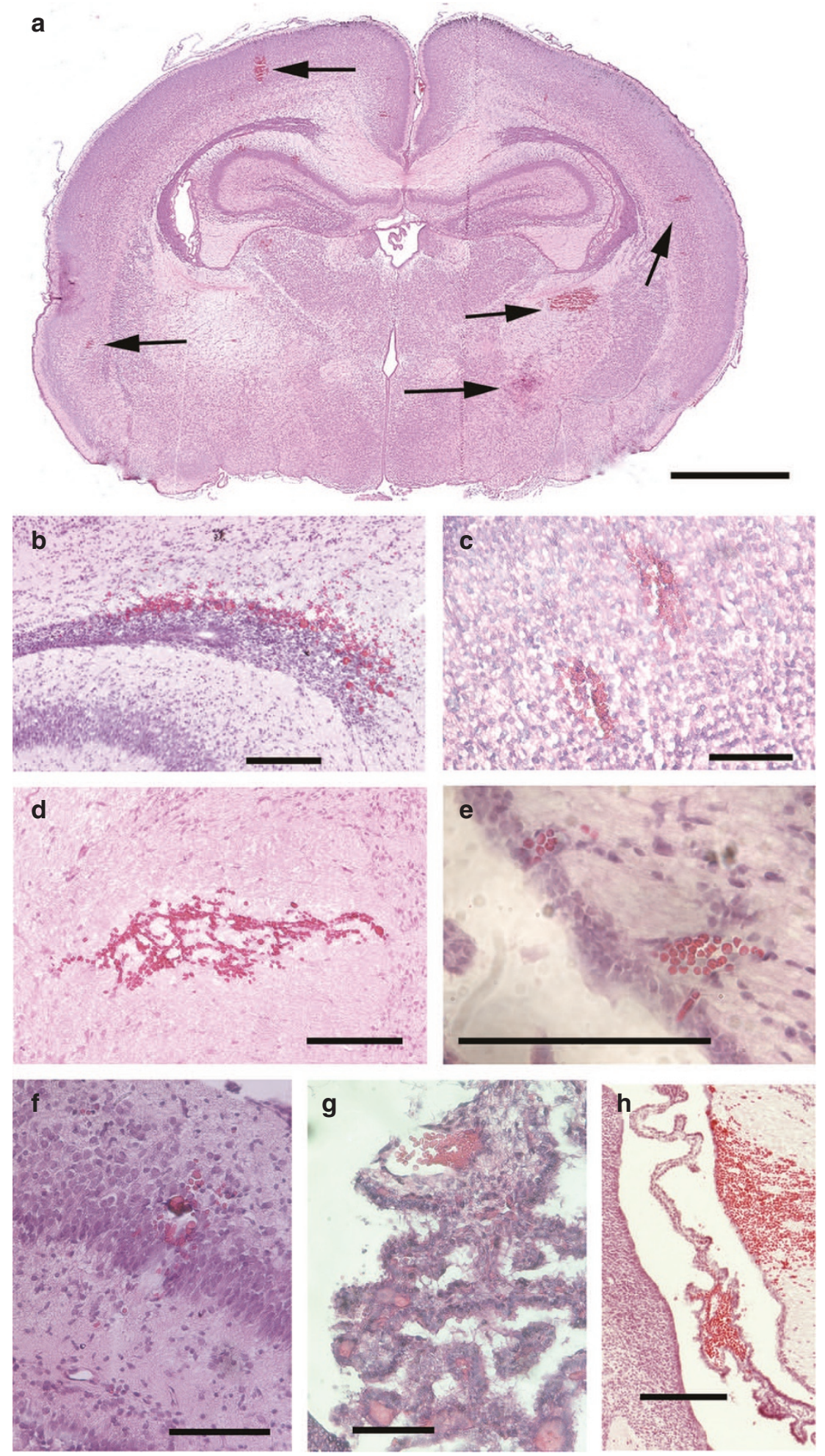

Figure 1. Periventricular hemorrhages in newborn rat pups. (a) Hematoxylin and eosin-stained coronal section of a brain $24 \mathrm{~h}$ after mechanical ventilation of a newborn pup that had been subjected to 20-min IUI 2-3 d previously (IUI+ventilation group); arrows point to hemorrhages. (b-h) Examples of hemorrhages in (b) subventricular zone, (c) cortex, (d) internal capsule, (e) periventricular vein with thrombosis, (f) hippocampus, (g) choroid plexus, and (h) lateral ventricle; scale bars: $1 \mathrm{~mm}$ in $\mathbf{a} ; 100 \mu \mathrm{m}$ in $\mathbf{b}-\mathbf{h}$.

newborn pups $2-5 \mathrm{~h}$ after birth, 2-3 d after IUI. In controls, collagen IV was least abundant in the cortex (Figure 3a), as reported (26). In fetuses examined within $24 \mathrm{~h}$ of IUI, we did not detect any difference in collagen IV expression by immunohistochemistry, compared to controls (sham-injury) (data not shown).

In pups examined 2-3 d after IUI, on P0 shortly after birth (no mechanical ventilation), we observed a significant reduction 


\section{Articles $\mid$ Tosun et al.}

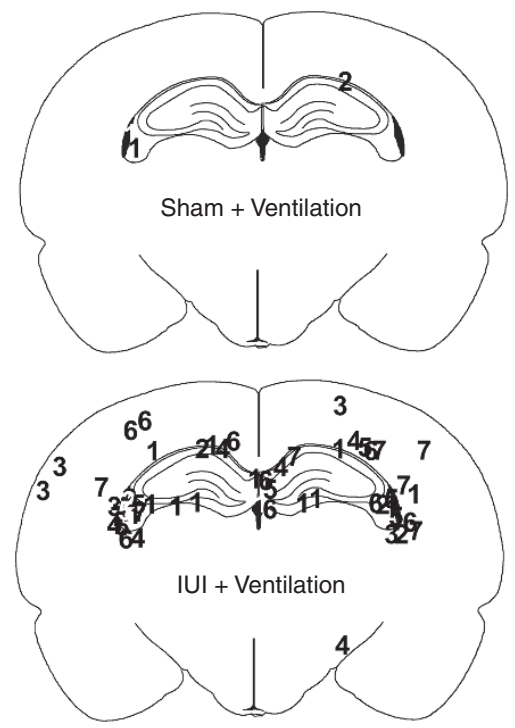

Figure 2. Distribution of periventricular hemorrhages in newborn rat pups. Maps showing the locations of hemorrhages identified $24 \mathrm{~h}$ after mechanical ventilation (a) in 2/7 pups that had been subjected to sham-injury or (b) in 7/7 pups that had been subjected to 20-min IUI 2 d previously (sham+ventilation vs. IUI+ventilation groups, respectively); hemorrhages in individual pups are identified by a number for that pup; data from seven pups per group.

of collagen IV in vessels, compared to controls (Figure 3a,b). We quantified collagen IV in three different areas: the internal capsule, periventricular area, and hippocampus. The most prominent reduction in collagen IV induced by IUI was in the periventricular area, with only $12 \%$ of that found in controls (Figure 3e). IUI also resulted in severe losses in collagen IV in the internal capsule and hippocampus.

Expression of the basement membrane protein, laminin, also was prominently reduced by IUI (Figure $4 c, d)$ ). Quantification revealed findings similar to those observed with collagen IV on P0 (Figure 3e).

\section{Angiogenesis: VEGF and MMP-9 upregulation after IUI}

Global ischemia in utero activates an angiogenic response in the brain that is most prominent in periventricular tissues (22). Fetuses were harvested at several times after IUI, and immunolabeling for VEGF was used to assess the angiogenic response. Compared to controls, VEGF-labeling of microvessels was robust as early as $3 \mathrm{~h}$ after ischemia, especially in periventricular regions. Quantifying the number of vessels that were immunopositive for VEGF showed a strong response at $3 \mathrm{~h}$ that diminished gradually during the first $24 \mathrm{~h}$ after IUI (Figure 4a-d).

The angiogenic response to ischemia involves not only VEGF upregulation but also MMP-9 upregulation, with MMP-9 potentially accounting for the loss of basement membrane collagen IV and laminin (27). Double labeling for VEGF and MMP-9 showed that both tended to colocalize (Figure 4e). Brain sections immunolabeled for MMP-9 showed specific labeling in microvessels $24 \mathrm{~h}$ post-IUI (Figure $5 \mathrm{a}-\mathrm{d}$ ). No MMP-9 expression was detected in sham-injured fetuses.
Upregulation of MMP-9 was not observed beyond $24 \mathrm{~h}$ after IUI (data not shown).

The transient upregulation of MMP-9 protein observed with immunolabeling was confirmed with immunoblots (Figure 5e). No change in abundance of MMP-9 was observed $3 \mathrm{~h}$ post-IUI. However, compared to sham-injury, there was a 1.8 -fold increase in MMP-9 protein in periventricular tissues from ischemic fetuses at $24 \mathrm{~h}$ after IUI.

To quantify the proteolytic activity of MMP-9, tissues were studied using zymography. Tissues from fetuses harvested 3,6 , and $12 \mathrm{~h}$ after IUI were not significantly different compared to sham-injury. However, strong bands corresponding to activated MMP-9 and MMP-2 were observed at $24 \mathrm{~h}$ (Figure 5f). Densitometric analysis revealed a 2.2 -fold increase in gelatinolytic activity. Similar to immunoblots, the enzymatic activity subsided after birth. Thus, $20 \mathrm{~min}$ of IUI was sufficient to prominently upregulate the expression and enzymatic activity of MMP-9 in periventricular areas $24 \mathrm{~h}$ later that accounted well for the delayed loss of collagen IV and laminin observed on P0.

\section{Inflammation}

Hemorrhages into the brain are known to induce an inflammatory response. The multiple periventricular hemorrhages induced in this model were expected to result in neuroinflammation associated with apoptotic neuronal death, as we previously observed (15). This was confirmed by immunolabeling for the activated microglial/macrophage marker, ED-1 (CD68), and for cleaved-caspase-3. Both ED-1 and cleaved-caspase-3 were prominently upregulated in the vicinity of the hemorrhages $24 \mathrm{~h}$ after mechanical ventilation in pups subjected to IUI, compared to sham-injured controls (Figure 6). The hippocampus was especially affected, but ED-1 positive cells also were found surrounding the hemorrhages in white matter tracts, including the internal and external capsule (Figure 6). Pups subjected to dual insults had significantly higher numbers of activated microglia and of cleaved caspase- 3 in periventricular areas, compared to sham-injured mechanically ventilated pups (Figure 6f).

\section{DISCUSSION}

The principal findings of the present study are (i) rat pups subjected to a prenatal ischemic insult were vulnerable to $\mathrm{PVH}$ when mechanically ventilated as newborns; (ii) the apparent reason for the increase in susceptibility to $\mathrm{PVH}$ was the loss of key basement membrane proteins required to maintain structural integrity under mechanical stress; (iii) the apparent reason for the loss of basement membrane proteins was upregulation/activation of MMP-9, as part of the angiogenic response to prenatal ischemia.

Here, we show that a 20 -min episode of mechanical ventilation after birth, a few days after prenatal ischemia, is causally related to PVH. Most pups that were ventilated exhibited lesions in the white matter surrounding the lateral ventricles, especially the internal capsule, as well as the subventricular zone, hippocampus and choroid plexus. Such hemorrhages 

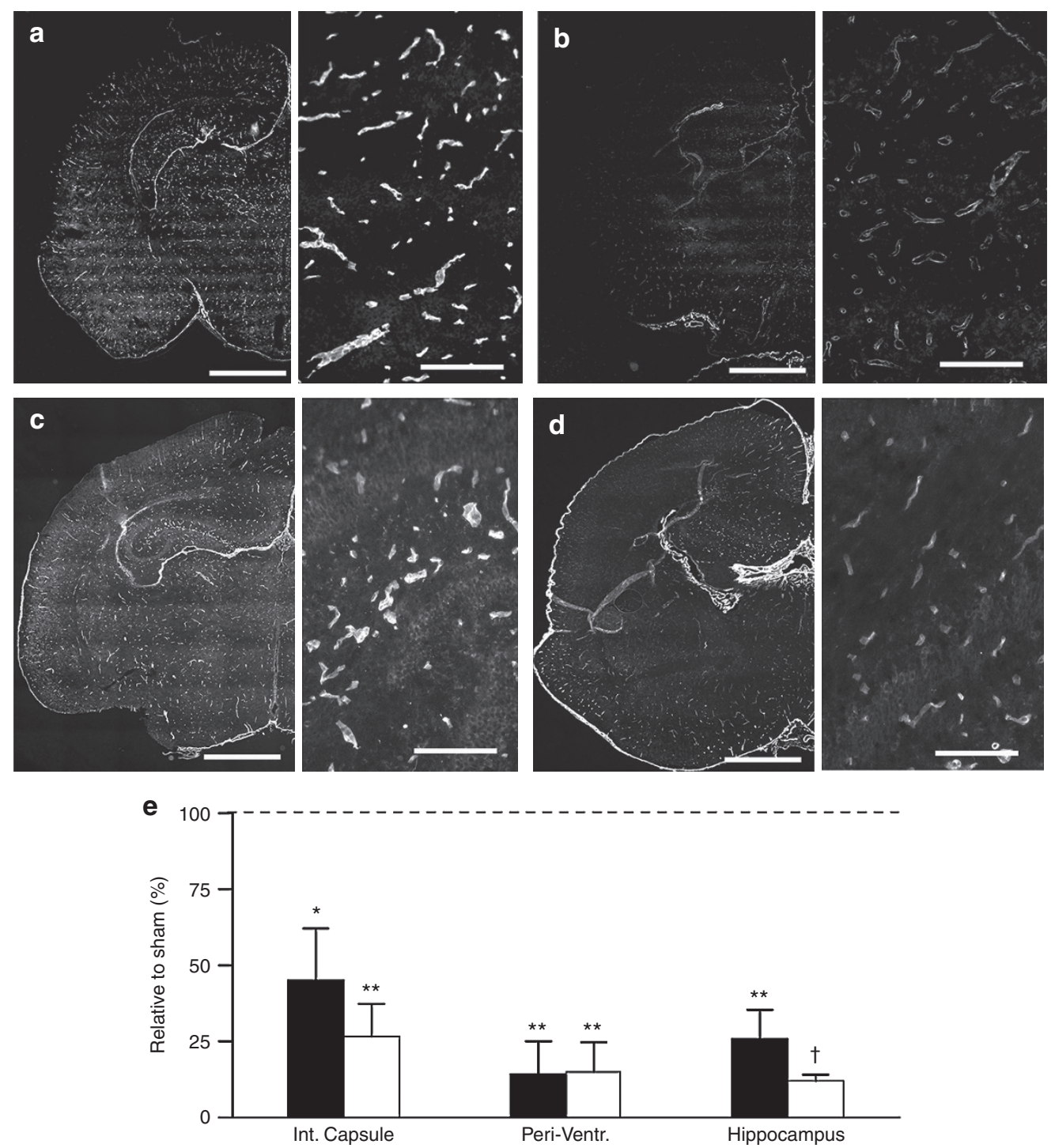

Figure 3. Loss of basement membrane components following prenatal ischemia. Immunolabeling for (a,b) collagen IV and (c,d) laminin in the brains of $(\mathbf{a}, \mathbf{c})$ a sham-injured pup and $(\mathbf{b}, \mathbf{d})$ a pup subjected to 20-min IUI $2 \mathrm{~d}$ previously; all tissues from P0 rats; each of the four panels shows a montage of a hemisphere (left) and a high power view of the hippocampus (right); scale bars: $1 \mathrm{~mm}$ and $50 \mu \mathrm{m}$ in a. (e) Quantitative analysis (mean \pm SE) of collagen IV (filled bars) and laminin (open bars) in the internal (int.) capsule, periventricular area (peri-vent), and hippocampus; data are expressed relative to values in sham-injured pups; seven pups per group; ${ }^{*} P<0.05 ;{ }^{* *} P<0.01{ }^{\dagger} P<0.001$; all images acquired at $20 \times$ magnification.

were not observed in mechanically ventilated pups without IUI (sham+ventilation group) or in nonventilated pups with IUI alone (14). Moreover, the marked inflammatory and celldeath responses, with activation of microglia/macrophages and activation of caspase- 3 that surrounded the ventilationinduced hemorrhages, underscore the severe pathological nature of these hemorrhagic lesions and their destructive capability. The incidence and distribution of the hemorrhages observed after mechanical ventilation were indistinguishable from the pattern observed in our previous experiments, which also incorporated 20-min IUI at the same prenatal age, but in which IP glycerol was used postnatally to transiently increase intravenous pressure. We showed previously that these parenchymal hemorrhages were venous in origin (14), and we showed that rats subjected to these tandem insults (IUI plus postnatal IP glycerol) later, as adolescents, exhibited significant developmental delay and abnormal cognitive and motor performance (15), recapitulating many findings in humans.

Angiogenesis-the formation of new blood vessels from preexisting vasculature-has been identified as a key factor predisposing to germinal matrix hemorrhage (12). Immature germinal matrix vessels have prominent ongoing angiogenic activity. In a premature rabbit model of germinal matrix hemorrhage, two angiogenic inhibitors, the cyclooxygenase-2 inhibitor, celecoxib, and the VEGF receptor 2 inhibitor, ZD6474, were found to significantly reduce susceptibility to hemorrhage (12). As reviewed by Ballabh et al. (8), selective vulnerability of angiogenic vessels may be due to an immature basal lamina, incomplete glial support or poor matrix support, all of which have been identified in both animal models and in premature infants. 


\section{Articles | Tosun et al.}
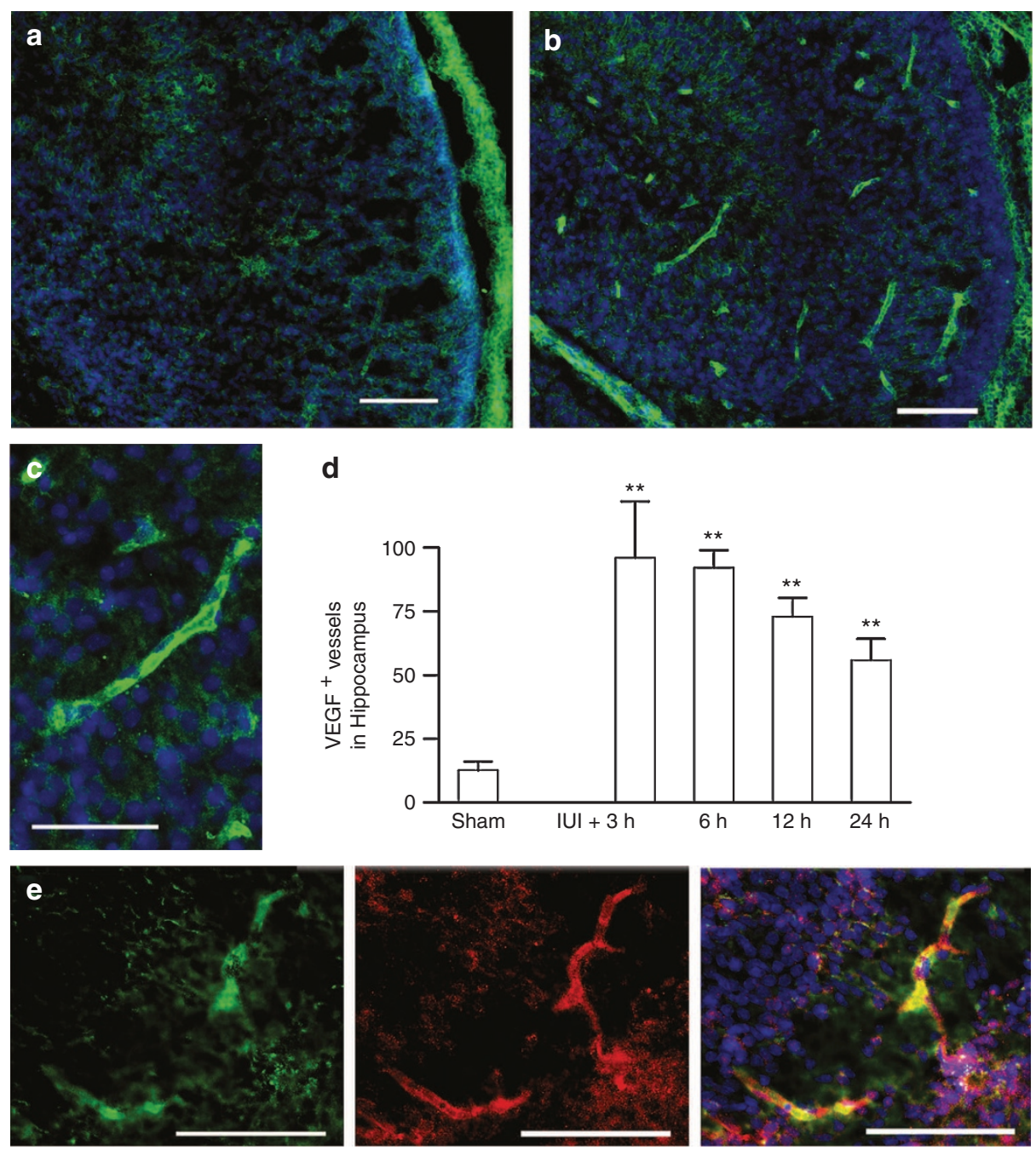

Figure 4. Angiogenesis induced by prenatal ischemia. Immunolabeling for vascular endothelial growth factor (VEGF) in the brains of (a) a sham-injured fetus and a fetus subjected to 20-min IUI $3 \mathrm{~h}$ previously, at (b) low and (c) high magnification; all tissues from E19 fetuses. (d) Quantitative analysis (mean $\pm \mathrm{SE}$ ) of the number of VEGF-positive microvessels in the hippocampus at various times after IUI or in sham-injured fetuses, as indicated; three fetuses per group. (e) Co-immunolabeling for VEGF (left; green) and MMP-9 (middle; red) in the brain of a fetus subjected to 20 -min IUI $24 \mathrm{~h}$ previously; merged images are shown (right; yellow); scale bars: $100 \mu \mathrm{m}$ in a and e; $50 \mu \mathrm{m}$ in c; ${ }^{* *} P<0.0$; all images acquired at $20 \times$ magnification.

Ischemic/hypoxic injury is a key trigger that induces angiogenesis in the brain $(22,28)$. The angiogenic response to ischemia/hypoxia involves the activation of the transcription factor, hypoxia inducible factor-1 (HIF-1), which is responsible for the transcriptional upregulation of numerous genes, including VEGF, angiopoitin-2, fibroblast growth factor and others, which are responsible for recruiting endothelial progenitor cells (EPCs) and promoting the formation of new vessels (29). The HIF-1-mediated angiogenic response also requires transciptional upregulation of enzymes responsible for proteolytic matrix degradation, which is needed for the migration of endothelial cells, EPCs and for vascular remodeling (18). During angiogenesis, degradation of the extracellular matrix is effected by matrix metalloproteinases (MMP), including the gelatinases, MMP-9, MMP-2 and others, which degrade the extracellular-matrix components of the basement membrane. VEGF upregulation and MMP-mediated degradation of the extracellular matrix are associated with increased permeability of the blood-brain barrier and edema formation (22).

Here, we found that the two essential markers of angiogenesis, VEGF and MMP-9, were prominently upregulated 3-24 h following IUI, prior to the development of hemorrhages. The maximum VEGF expression was found at $3 \mathrm{~h}$, whereas the maximum gelatinase activity of MMP-9 was identified at $24 \mathrm{~h}$. Angiogenesis, as signaled by VEGF upregulation, recently was reported in sheep following 10-min IUI (22). As shown here and previously by us $(14,15)$ and others $(22,30)$, a prenatal ischemic insult is associated with microvascular changes principally in periventricular regions, possibly due to the "endarteriolar" vascular supply of these regions.

The major components of the extracellular matrix of the cerebral vasculature, including laminin, fibronectin, collagen $\mathrm{IV}$, and perlecan (31), provide structural stability to blood vessels that is especially important during mechanical stress. Both collagen IV and laminin were markedly degraded $2-3 \mathrm{~d}$ after 

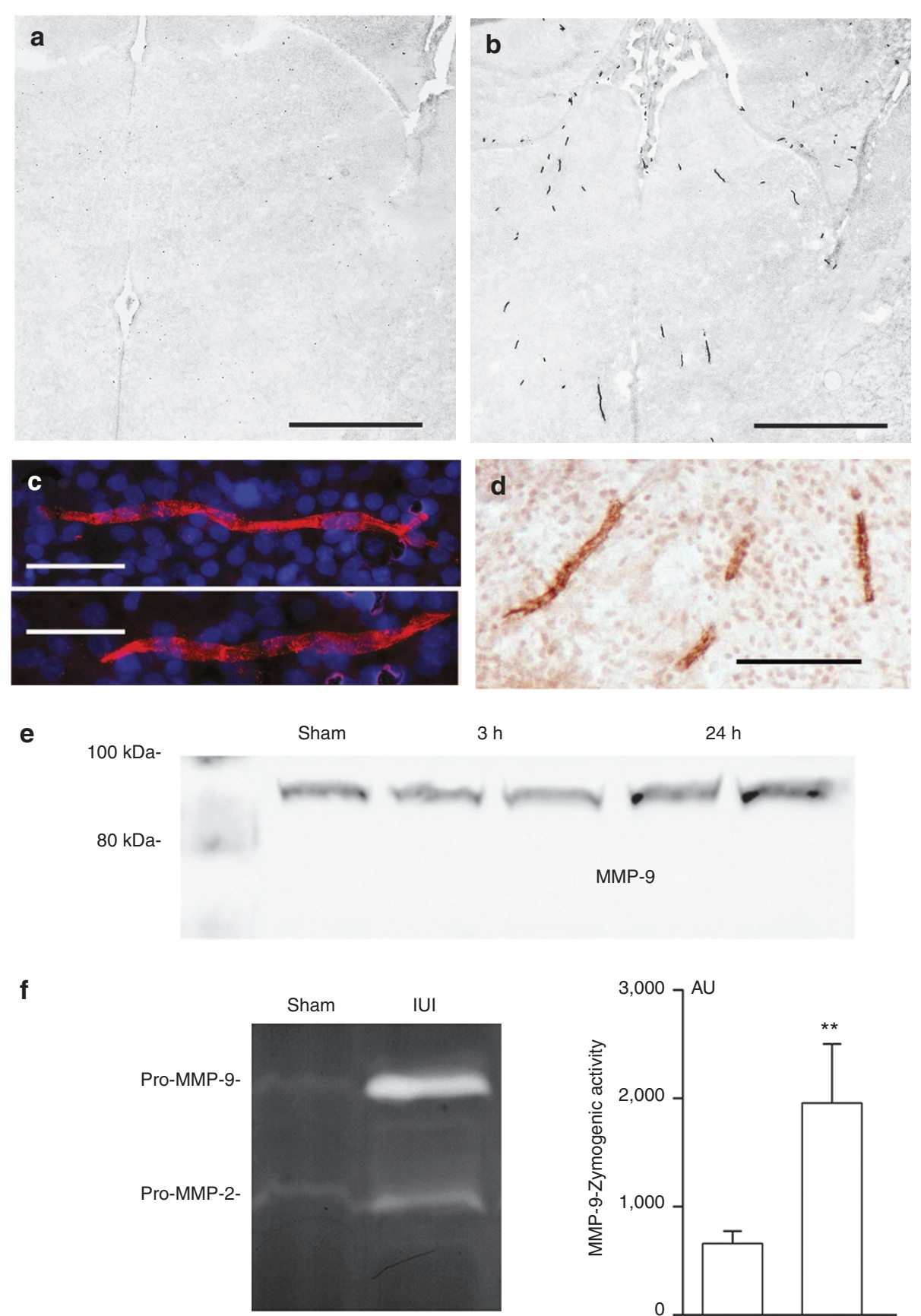

$3 \mathrm{~h}$

$24 \mathrm{~h}$

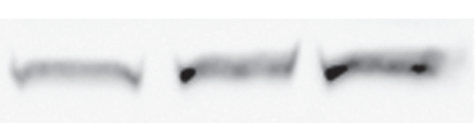

MMP-9

Figure 5. Matrix metalloproteinase-9 induced by prenatal ischemia. Immunolabeling for MMP-9 in the brains of (a) a sham-injured fetus and (b-d) a fetus subjected to 20-min IUI $24 \mathrm{~h}$ previously, at (b) low and (c,d) high magnification, using (a-c) fluorescent secondary antibody or (d) chromagen for visualization; in $\mathbf{a}$ and $\mathbf{b}$, inverted black/white images are shown for clarity; scale bar: $1 \mathrm{~mm}$ in a, $50 \mu \mathrm{m}$ in c, $100 \mu \mathrm{m}$ in d. (e) Immunoblot for MMP-9 of brain lysates from sham-injury and 3 or $24 \mathrm{~h}$ after IUI; 2 fetuses for both times after IUI. (f) Zymography showing MMP-9 and MMP-2 activity in brain lysates from sham and $24 \mathrm{~h}$ after IUI (left); quantitative analysis (mean $\pm \mathrm{SE}$ ) of zymography for MMP-9 activity; ${ }^{* *} P<0.01$; five fetuses per group; all images acquired at $20 \times$ magnification.

IUI. Defects in collagen IV, laminin, and fibronectin in choroid plexus and immature subependymal microvessels previously have been observed in preterm neonatal humans with germinal matrix hemorrhages (32). Similarly, a poorly developed basal lamina and a paucity of collagen in germinal matrix vessels are believed to predispose to germinal matrix hemorrhage in premature rabbits (13). Of the many components that constitute the extracellular matrix, collagen IV plays an especially crucial role. Mutation of Col4a1, the gene that encodes collagen IV, is sufficient by itself to yield exquisitely fragile vessels leading to widespread spontaneous perinatal brain hemorrhages (25). No other matrix component has been reported to play such a pivotal role.

An important shortcoming of the present work is that we did not explicitly demonstrate the requirement for MMP-9. We showed using immunoblot, immunohistochemistry and 


\section{Articles $\mid$ Tosun et al.}
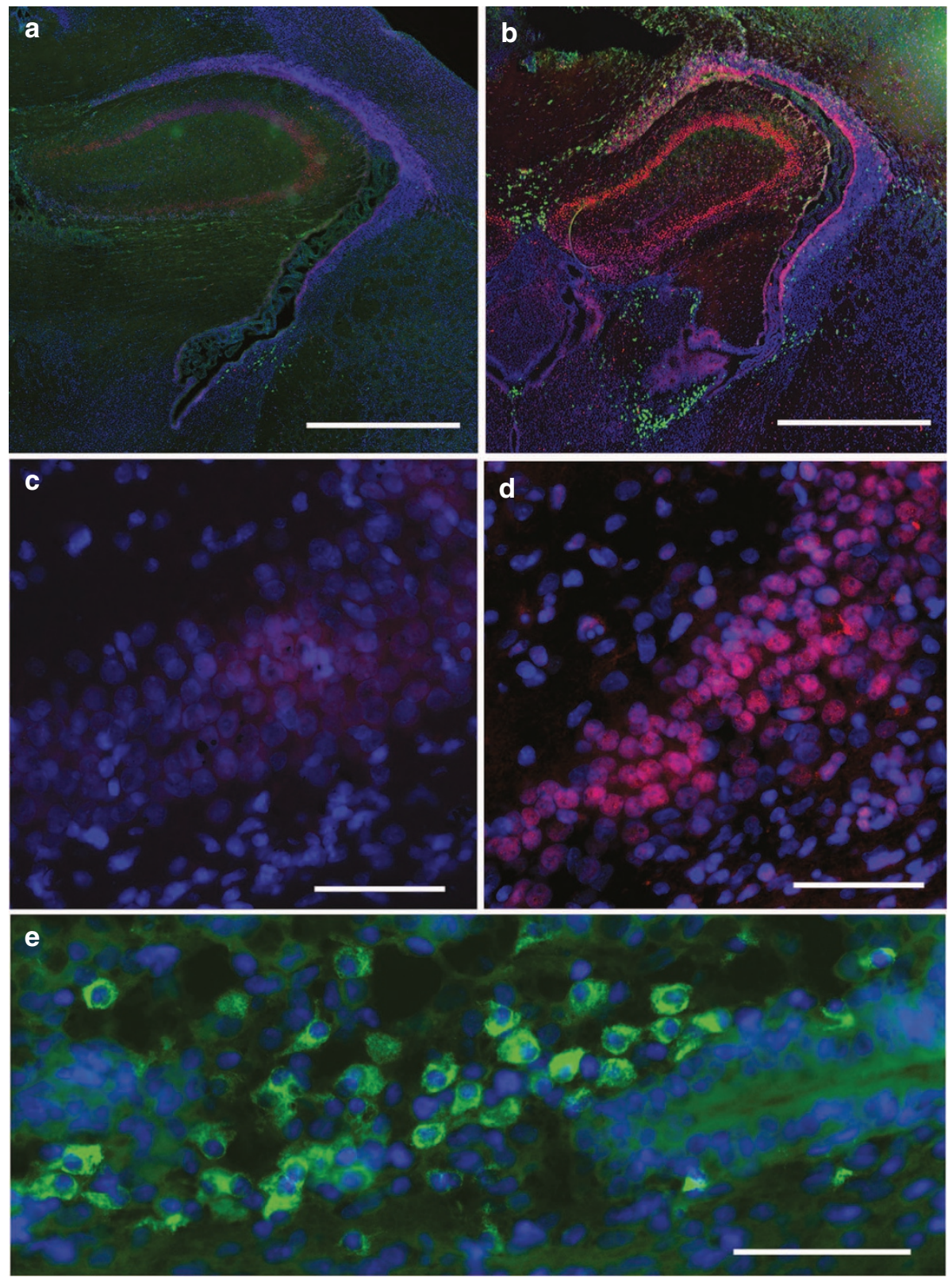
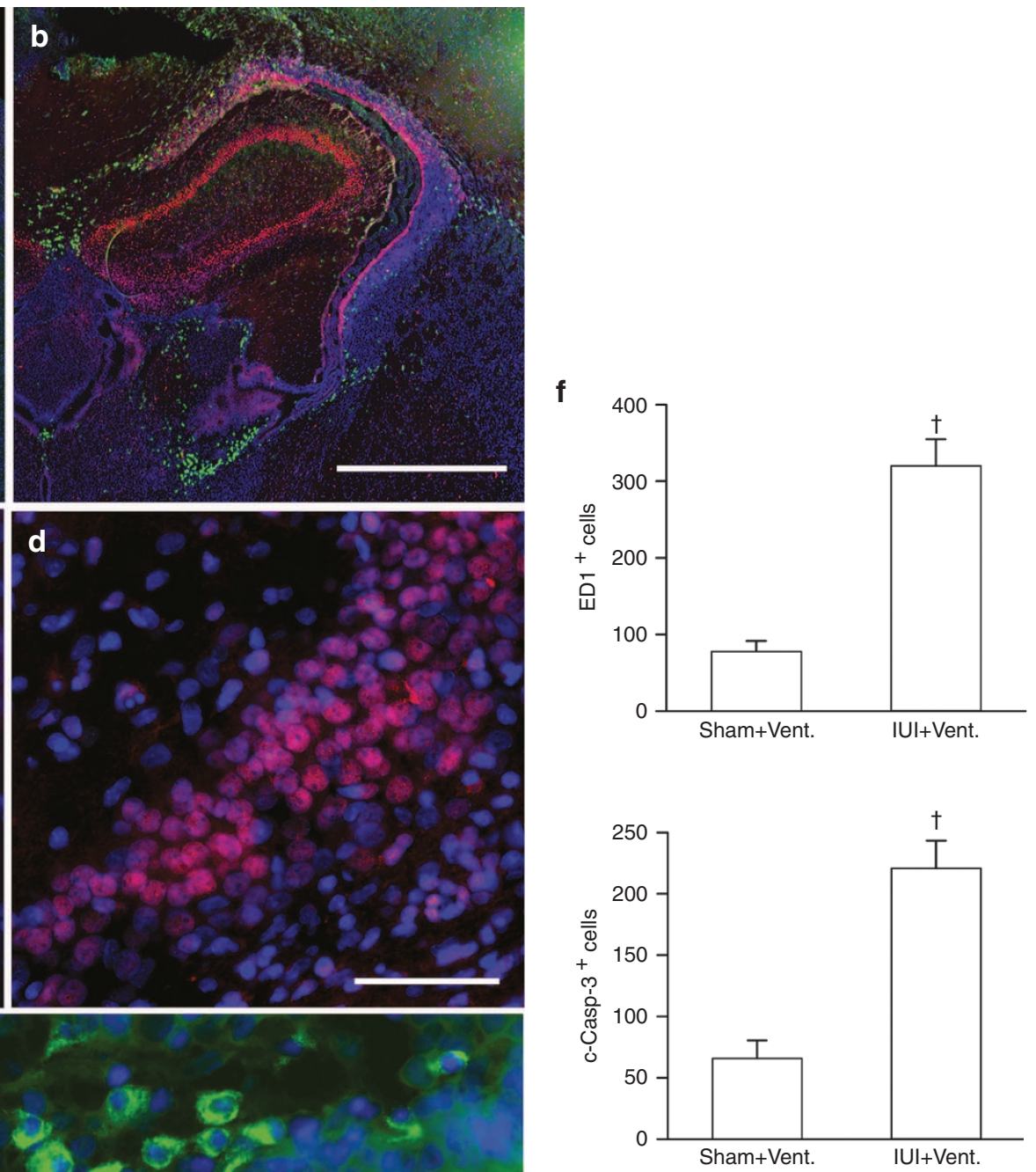

Figure 6. Periventricular neuroinflammation and cell death induced by the dual insults of prenatal ischemia and postnatal mechanical ventilation. (a-e) Immunolabeling for the marker of apoptosis, activated caspase-3 (red), and the marker of activated microglia/macrophages, ED-1 (green) in the brains of $(\mathbf{a}, \mathbf{c})$ a sham+ventilation pup and $(\mathbf{b}, \mathbf{d}, \mathbf{e})$ an IUI+ventilation pup; the images in $\mathbf{c}$ and $\mathbf{d}$ are high magnification views of the hippocampal dendate gyri shown in $\mathbf{a}$ and $\mathbf{b}$; the image in $\mathbf{e}$ is a high power view of the periventricular area below the fimbria in $\mathbf{b}$; all tissues from P1 rats. (f) Quantitative analysis (mean \pm SE) of ED-1-positive cells (left panel) and of cleaved caspase-3-positive cells (right panel) in sham+ventilation vs. IUI+ventilation pups; 5-7 pups per group; ${ }^{\dagger} P<0.001$; images in $\mathbf{a}$ and $\mathbf{b}$ acquired at $20 \times$ magnification, those in $\mathbf{c}-\mathbf{e}$ acquired at $40 \times$ magnification.

zymography that brain MMP-9 was significantly upregulated/ activated by IUI, and we showed that the MMP-9 substrates, collagen IV and laminin, were significantly degraded following the appearance and activation of MMP-9. However, experiments specifically to block MMP-9 activity were not performed. Pharmacological MMP-9 inhibitors are available (33), and have been shown to be beneficial in neonatal ischemia/hypoxia (Rice Vannucci model) (20). However, their use in the context of IUI has not been reported, their ability to cross the placental barrier is not known, and direct in utero administration is not practical. Genetic deletion of MMP-9 also could be considered, as these mice are available. However, we have found that IUI of pregnant mice carries high maternal mortality, and mechanical ventilation of newborn mice is not yet feasible. Further work will be required to establish what we hypothesize to be the key role of MMP-9 in models of hemorrhagic-EP.

In summary, we showed a causal relationship between IUI, mechanical ventilation and periventricular hemorrhages within our experimental paradigm. We demonstrated that collagen, the most important basement membrane protein required for vessel integrity, was severely degraded following 


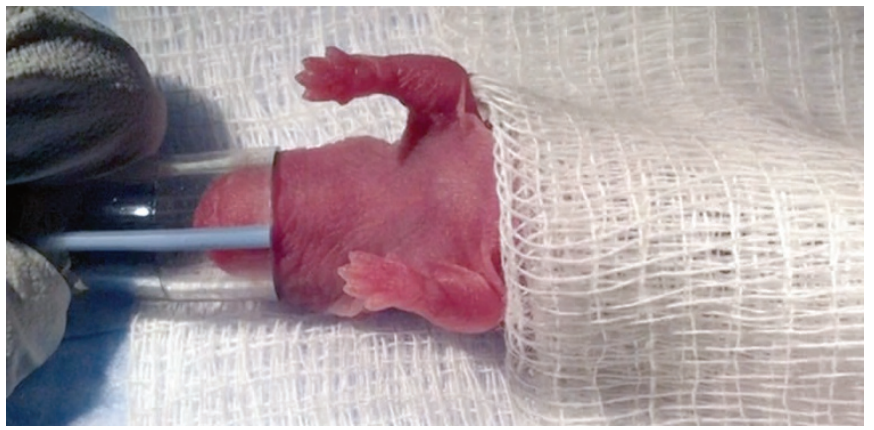

Figure 7. Mechanical ventilation of a newborn rat pup using a face mask. The snout fits snugly inside the $2-\mathrm{cm}$ long segment of soft polyvinyl tubing (I.D., $8 \mathrm{~mm}$ ) used a as face mask, without occluding the mouth or nares. The face mask is connected to a mechanical ventilator (see Methods).

IUI, a finding that provides a strong basis for understanding the observed vulnerability to mechanical ventilation induced by IUI. We also expanded on these observations by speculating on the role of MMP-9 upregulation/activation, which is a natural part of the angiogenic response to hypoxia. Our experiments represent a novel advancement of the work of Ballabh et al. (12), who showed that constitutive angiogenesis predisposes to hemorrhage in the germinal matrix-here, we showed that hypoxia-induced angiogenesis in utero can predispose to hemorrhages in periventricular tissues. Our findings underscore the importance of inducing ischemia in the premature rodent brain prenatally (i.e., IUI), rather than in the mature rodent postnatally (i.e., Rice Vannucci model), when modeling PVH. Our data with our tandem insult models reported here and previously $(14,15)$ suggest that in regions of the developing brain undergoing hypoxia-induced angiogenesis, transient degradation of the extracellular matrix may leave these regions susceptible to hemorrhage during periods of mechanical stress, as can occur with raised intravenous pressure or mechanical ventilation.

\section{METHODS}

\section{Ethics statement}

Animal experiments were performed under a protocol approved by the Institutional Animal Care and Use Committee of the University of Maryland, and in accordance with the guidelines and regulations in the NIH Guide for the Care and Use of Laboratory Animals.

\section{In utero ischemia and mechanical ventilation}

IUI was induced as described (14). On E19, timed pregnant Wistar rats (Harlan, Frederick, MD) underwent laparotomy to externalize the uterus. The ovarian and uterine blood supplies were clamped for $20 \mathrm{~min}$, after which the uterus was reinternalized. For sham-injury, the same procedures were performed, but the vasculature was not clamped. In some experiments (series 1 and series 2), the dam gave birth to pups naturally, $\sim 2-3 \mathrm{~d}$ following IUI (postnatal day 0 (P0)), whereas in other experiments (series 3 ), fetuses were harvested by Caesarean section at various times after IUI.

Mechanical ventilation was carried out with room air without anesthesia on $\mathrm{P} 0,6 \mathrm{~h}$ after natural birth. The respiratory rate of newborn rats is $\sim 150$ breaths/min (bpm) (34). Previous studies used moderate tidal volumes $\left(\mathrm{V}_{\mathrm{T}}\right)$ of $8.5-10 \mathrm{ml} / \mathrm{kg}(34,35)$. We adjusted the ventilator stroke volume based on the weight of each pup to obtain $\mathrm{V}_{\mathrm{T}}=$ $10 \mathrm{ml} / \mathrm{kg}$. The duration of mechanical ventilation was $20 \mathrm{~min}$. A face mask was constructed from a $2-\mathrm{cm}$ long segment of polyvinyl tubing (I.D., $8 \mathrm{~mm}$ ) and connected to a Harvard rodent ventilator (model 683, adjusted for small tidal volumes; Harvard Apparatus, Holliston,
Table 2. Number of subjects in each experimental series

\begin{tabular}{cccl}
\hline & Sham & Ventilation & \multicolumn{1}{c}{ Experiment } \\
\hline series 1 & 10 & 18 & $\begin{array}{l}\text { Blood gases and electrolytes } \\
\text { at P0 }\end{array}$ \\
Sham+Vent & IUI+Vent & \\
& 7 & 7 & H\&E for hemorrhage at P1 \\
& 5 & 7 & ED-1 and caspase-3 at P1 \\
series 3 & SHAM & IUI & \\
& 3 & 3/time point & $\begin{array}{l}\text { Collagen, laminin, VEGF, MMP-9 } \\
\text { at 3, 6, 12, and 24 h post-IUI }\end{array}$ \\
& 2 & 2/time point & $\begin{array}{l}\text { MMP-9 immunoblot at 3 and } \\
24 \mathrm{~h} \text { post-IUI }\end{array}$ \\
& 5 & 5/time point & $\begin{array}{l}\text { MMP-9 zymography at 3, 6, 12, } \\
\text { 24h post-IUI } \\
\end{array}$ \\
& 7 & 7 & Collagen and laminin at P0
\end{tabular}

IUI, intrauterine ischemia; Vent, mechanical ventilation.

MA). The face mask was secured to the snout by hand to prevent air leakage (Figure 7). Body temperature was maintained using a heating pad and a gauze sponge. Time away from the dam was minimized.

\section{Experimental series}

Pups in series 1 were used for the analysis of blood gases and electrolytes immediately after mechanical ventilation (Table 2). In series 2 , newborn pups that had been subjected to IUI and mechanical ventilation after birth comprised the IUI+ventilation group; newborn pups that had been subjected to sham-injury in utero and mechanical ventilation after birth comprised the sham+ventilation (control) group (Table 2). Pups in series 2 were examined for intracerebral hemorrhages, or ED-1 and cleaved caspase- 3 at P1.

In series 3, fetuses were euthanized at various times after IUI or after sham-injury (Table 2). To remove intravascular blood, fetuses were individually perfused with ice-cold normal saline (NS) under microscopic visualization while the dam was under anesthesia. Some of these brains were flash frozen in liquid nitrogen, kept at $-80{ }^{\circ} \mathrm{C}$ and were used for immunoblotting and zymography. For the other brains, NS perfusion was followed by perfusion with $4 \%$ paraformaldehyde (PFA), and these brains were used for immunolabeling.

\section{Blood gas and electrolyte analysis}

Pups in series 1 were decapitated immediately after completing 20 min mechanical ventilation to obtain mixed arterial/venous blood. Blood was collected in an Eppendorf tube containing ice-cold buffered sodium citrate $(0.109 \mathrm{M})$ (Becton, Dickinson and Company, Franklin Lakes, NJ) (24). Samples were placed on ice and were analyzed immediately using an Element POC blood gas \& electrolyte analyzer (Heska, Loveland, CO). The time between the collection and delivery to the analyzer was $<30 \mathrm{~s}$ for each sample.

\section{Brain hemorrhages}

Pups in series 2 were euthanized $24 \mathrm{~h}$ after birth (P1), intravascular blood was removed by perfusing with ice-cold NS, and brains were examined for intracerebral hemorrhages. Coronal sections at the level of the third ventricle were labeled with hematoxylin and eosin (H\&E). The locations of the hemorrhages on brain sections were mapped onto an atlas image, superimposing the locations of all hemorrhages for each pup in a given treatment group (sham+ventilation vs. IUI+ventilation).

\section{Immunohistochemistry}

The brains of some pups in series 2 and fetuses in series 3 that had been perfused with $4 \%$ PFA were postfixed overnight in PFA. Brains were transferred to $30 \%$ sucrose for cryopreservation then frozen in OCT. Cryosections $(10 \mu \mathrm{m})$ were blocked with $2 \%$ donkey serum with $0.2 \%$ Triton X-100 in $1 \times$ phosphate buffered saline (PBS) for $1 \mathrm{~h}$ at room temperature (RT). Sections were incubated overnight with primary antibodies, including: rabbit anti-collagen IV (1:400; ab6586; Abcam, 
Cambridge, MA); FITC-conjugated rabbit anti-laminin (1:500; FA-2404-1; EY Labs, San Mateo, CA); rabbit anti-MMP-9 (1:200; ab38898; Abcam); rabbit anti-VEGF-A20 (1:200; sc-152; Santa Cruz Biotechnology, Dallas, TX); rabbit anti-cleaved caspase-3-Asp175 (1:200; 9661; Cell Signaling Technology, Danvers, MA); mouse antimacrophages/monocytes (ED-1) (1:200; MAB 1435; Merk Millipore; Billerica, MA) at $4{ }^{\circ} \mathrm{C}$. After several rinses in PBS, sections were incubated with species-appropriate fluorescent secondary antibodies (Alexa Flour 488 and 555, Molecular Probes, Invitrogen, Carlsbad, CA) for $1 \mathrm{~h}$ at room temperature. Controls included the omission of primary antibodies.

Biotin-conjugated secondary antibodies were used for chromogen immunolabeling. Sections were incubated in $0.3 \% \mathrm{H}_{2} \mathrm{O}_{2}$ for $30 \mathrm{~min}$. After three washes in PBS, sections were blocked with $0.2 \%$ Triton X-100 (Sigma-Aldrich, St Louis, MO) in PBS containing 5\% goat serum (Sigma-Aldrich) for $1 \mathrm{~h}$ at RT, then incubated overnight with anti-MMP-9 antibody (1:1,000 in blocking solution). The next day, sections were washed in PBS and incubated with biotinylated secondary antibody (BA-1000 1:500 goat anti-rabbit; Vector Laboratories, Burlingame, CA) for $2 \mathrm{~h}$. After washing in PBS, sections were incubated in avidin-biotin solution (Vector Laboratories) and the color was developed in diaminobenzidine chromogen solution $(0.02 \%$ diaminobenzidine in $0.175 \mathrm{M}$ sodium acetate) activated with $0.01 \%$ hydrogen peroxide. Cresyl violet was used as a counterstain.

\section{Quantification of immunofluorescence}

Unbiased measurements of signal intensity within regions of interest (ROI) were obtained using NIS-Elements AR software (Nikon Instruments, Melville, NY) from sections immunolabeled in a single batch. All ROI images for a given signal were captured using uniform parameters of magnification, area, exposure, and gain. Segmentation analysis was performed by computing a histogram of pixel intensity for a particular ROI. For collagen IV and laminin, specific labeling was defined as pixels with signal intensity greater than $3 \times$ that of background; the area occupied by pixels with specific labeling was used to determine the percent area with specific labeling (\% ROI). For VEGF, ED-1, and activated caspase-3, specific labeling was defined as above, and object counts within the ROI were performed. For collagen IV and laminin, oval-shaped ROIs were located at the internal capsule (width $\times$ height, $300 \times 600 \mu \mathrm{m}$ ), periventricular area $(1,000 \times 300 \mu \mathrm{m})$, and hippocampus $(500 \times 1200 \mu \mathrm{m})$. For VEGF, an oval-shaped ROI was located in the hippocampus $(500 \times 1,000 \mu \mathrm{m})$. For ED-1, the ROI was defined as a perimeter $200 \mu \mathrm{m}$ from the lateral ventricles. For activated caspase-3, the ROI included the entire granular layer of CA3.

\section{Western blotting}

The brains of fetuses from series 3 that had been flash frozen were used. The cerebrum was divided coronally into three equal pieces, and the middle part was homogenized in buffer $(0.1 \%$ Triton X-100 in $1 \times$ PBS with protease inhibitors) on ice; $35 \mu \mathrm{g}$ of total protein was loaded onto $3-8 \%$ tris-acetate gels, and membranes were blotted with antibodies against MMP-9 (1:1000 in 5\% BSA, Millipore, Billerica, MA) overnight at $4{ }^{\circ} \mathrm{C}$. Blots were quantified using Image J software (National Institutes of Health, Bethesda, MD) and normalized to actin as a loading control.

\section{Zymography}

The brains of fetuses from series 3 that had been flash frozen were used. The cerebrum was divided coronally into three equal pieces, and the middle part was homogenized. Zymography was carried out using Novex Zymogram kits (Life Technologies, Grand Island, NY), used in accordance with the manufacturer's protocol. The activity of MMP-9 was quantified using Image J software.

\section{Data analysis}

Data are presented as mean \pm SE. Statistical analyses (unpaired $t$-test) were performed using Origin Pro (V8; OriginLab, North Hampton, MA).

\section{STATEMENT OF FINANCIAL SUPPORT}

This work was supported by grants to J.M.S. from the US National Institutes of Health, including the National Heart, Lung and Blood Institute (HL082517) and the National Institute of Neurological Disorders and Stroke (NS061808). Disclosure: There is no conflict of interest to disclose.

\section{REFERENCES}

1. Volpe JJ. Brain injury in premature infants: a complex amalgam of destructive and developmental disturbances. Lancet Neurol 2009;8:110-24.

2. Kinney HC, Volpe JJ. Modeling the encephalopathy of prematurity in animals: the important role of translational research. Neurol Res Int 2012;2012:295389.

3. Raybaud C, Ahmad T, Rastegar N, Shroff M, Al Nassar M. The premature brain: developmental and lesional anatomy. Neuroradiology 2013;55 Suppl 2:23-40.

4. Futagi Y, Toribe Y, Ogawa K, Suzuki Y. Neurodevelopmental outcome in children with intraventricular hemorrhage. Pediatr Neurol 2006;34:21924.

5. Ou X, Glasier CM, Ramakrishnaiah RH, et al. Impaired white matter development in extremely low-birth-weight infants with previous brain hemorrhage. AJNR Am J Neuroradiol 2014;35:1983-9.

6. Pavlova MA, Krägeloh-Mann I. Limitations on the developing preterm brain: impact of periventricular white matter lesions on brain connectivity and cognition. Brain 2013;136(Pt 4):998-1011.

7. Bloch JR. Antenatal events causing neonatal brain injury in premature infants. J Obstet Gynecol Neonatal Nurs 2005;34:358-66.

8. Ballabh P. Intraventricular hemorrhage in premature infants: mechanism of disease. Pediatr Res 2010;67:1-8.

9. Tsai WH, Hwang YS, Hung TY, Weng SF, Lin SJ, Chang WT. Association between mechanical ventilation and neurodevelopmental disorders in a nationwide cohort of extremely low birth weight infants. Res Dev Disabil 2014;35:1544-50.

10. Thomas CW, Meinzen-Derr J, Hoath SB, Narendran V. Neurodevelopmental outcomes of extremely low birth weight infants ventilated with continuous positive airway pressure vs. mechanical ventilation. Indian J Pediatr 2012;79:218-23.

11. Van Marter LJ, Kuban KC, Allred E, et al.; ELGAN Study Investigators. Does bronchopulmonary dysplasia contribute to the occurrence of cerebral palsy among infants born before 28 weeks of gestation? Arch Dis Child Fetal Neonatal Ed 2011;96:F20-9.

12. Ballabh $\mathrm{P}, \mathrm{Xu} \mathrm{H}, \mathrm{Hu} F$, et al. Angiogenic inhibition reduces germinal matrix hemorrhage. Nat Med 2007;13:477-85.

13. Sotrel A, Lorenzo AV. Ultrastructure of blood vessels in the ganglionic eminence of premature rabbits with spontaneous germinal matrix hemorrhages. J Neuropathol Exp Neurol 1989;48:462-82.

14. Koltz MT, Tosun C, Kurland DB, et al. Tandem insults of prenatal ischemia plus postnatal raised intrathoracic pressure in a novel rat model of encephalopathy of prematurity. J Neurosurg Pediatr 2011;8:628-39.

15. Tosun C, Koltz MT, Kurland DB, et al. The protective effect of glibenclamide in a model of hemorrhagic encephalopathy of prematurity. Brain Sci 2013;3:215-38.

16. Sternlicht MD, Werb Z. How matrix metalloproteinases regulate cell behavior. Annu Rev Cell Dev Biol 2001;17:463-516.

17. Asahi M, Wang X, Mori T, et al. Effects of matrix metalloproteinase-9 gene knock-out on the proteolysis of blood-brain barrier and white matter components after cerebral ischemia. J Neurosci 2001;21:7724-32.

18. Morancho A, Hernández-Guillamon $\mathrm{M}$, Boada $\mathrm{C}$, et al. Cerebral ischaemia and matrix metalloproteinase- 9 modulate the angiogenic function of early and late outgrowth endothelial progenitor cells. J Cell Mol Med 2013; 17:1543-53.

19. Bednarek N, Svedin P, Garnotel R, et al. Increased MMP-9 and TIMP-1 in mouse neonatal brain and plasma and in human neonatal plasma after hypoxia-ischemia: a potential marker of neonatal encephalopathy. Pediatr Res 2012;71:63-70.

20. Chen W, Hartman R, Ayer R, et al. Matrix metalloproteinases inhibition provides neuroprotection against hypoxia-ischemia in the developing brain. J Neurochem 2009;111:726-36.

21. Svedin P, Hagberg H, Sävman K, Zhu C, Mallard C. Matrix metalloproteinase- 9 gene knock-out protects the immature brain after cerebral hypoxiaischemia. J Neurosci 2007;27:1511-8. 
22. Baburamani AA, Castillo-Melendez M, Walker DW. VEGF expression and microvascular responses to severe transient hypoxia in the fetal sheep brain. Pediatr Res 2013;73:310-6.

23. Pellicer A, Gayá F, Madero R, Quero J, Cabañas F. Noninvasive continuous monitoring of the effects of head position on brain hemodynamics in ventilated infants. Pediatrics 2002;109:434-40.

24. Vaillancourt C, Berger N, Boksa P. Effects of vaginal birth versus caesarean section birth with general anesthesia on blood gases and brain energy metabolism in neonatal rats. Exp Neurol 1999;160:142-50.

25. Gould DB, Phalan FC, Breedveld GJ, et al. Mutations in Col4al cause perinatal cerebral hemorrhage and porencephaly. Science 2005;308:1167-71.

26. Anstrom JA, Thore CR, Moody DM, Challa VR, Block SM, Brown WR. Morphometric assessment of collagen accumulation in germinal matrix vessels of premature human neonates. Neuropathol Appl Neurobiol 2005;31:181-90.

27. Van den Steen PE, Dubois B, Nelissen I, Rudd PM, Dwek RA, Opdenakker G. Biochemistry and molecular biology of gelatinase B or matrix metalloproteinase-9 (MMP-9). Crit Rev Biochem Mol Biol 2002;37:375-536.

28. Virgintino D, Girolamo F, Rizzi M, et al. Ischemia/Reperfusion-induced neovascularization in the cerebral cortex of the ovine fetus. J Neuropathol Exp Neurol 2014;73:495-506.
29. Fan X, Heijnen CJ, van der Kooij MA, Groenendaal F, van Bel F. The role and regulation of hypoxia-inducible factor-1alpha expression in brain development and neonatal hypoxic-ischemic brain injury. Brain Res Rev 2009;62:99-108.

30. Baburamani AA, Lo C, Castillo-Melendez M, Walker DW. Morphological evaluation of the cerebral blood vessels in the late gestation fetal sheep following hypoxia in utero. Microvasc Res 2013;85:1-9.

31. Xu H, Hu F, Sado Y, et al. Maturational changes in laminin, fibronectin, collagen IV, and perlecan in germinal matrix, cortex, and white matter and effect of betamethasone. J Neurosci Res 2008;86:1482-500.

32. Wei W, Xin-Ya S, Cai-Dong L, Zhong-Han K, Chun-Peng C. Relationship between extracellular matrix both in choroid plexus and the wall of lateral ventricles and intraventricular hemorrhage in preterm neonates. Clin Anat 2000;13:422-8.

33. Kaczmarek L. Mmp-9 inhibitors in the brain: can old bullets shoot new targets? Curr Pharm Des 2013;19:1085-9.

34. Iben SC, Haxhiu MA, Farver CF, Miller MJ, Martin RJ. Short-term mechanical ventilation increases airway reactivity in rat pups. Pediatr Res 2006;60:136-40.

35. Kroon AA, Wang J, Kavanagh BP, et al. Prolonged mechanical ventilation induces cell cycle arrest in newborn rat lung. PLoS ONE 2011;6:e16910. 